Recepción: 20 / 04 / 2017

Aceptación: 20 / 05 / 2017

Publicación: 15 / 07 / 2017

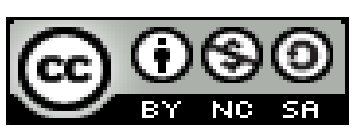

Ciencias técnicas y aplicadas

Artículo Científico

\title{
Procesos logísticos en el seguimiento de contenedores por vía terrestre desde la liberación de las almaceneras
}

\author{
Logistic processes in the tracking of containers by land since the release of the \\ warehouses
}

Processos logísticos no rastreamento de contêineres por terra desde a liberação dos armazéns

\author{
Andrea E. Villacis-Ramírez ${ }^{\text {I }}$ \\ avillacisra@gmail.com \\ Ana J. Haro-Velastegui II \\ anahvelastegui@gmail.com \\ Henry F. Lavayen-Yavar III \\ henryylavayen@gmail.com
}

Correspondencia: avillacisra@gmail.com

\footnotetext{
I. Magister en Administración de Empresas con Mención en Logística y Transporte; Ingeniera en Gestión Empresarial Internacional; Universidad de Guayaquil, Guayaquil, Ecuador.

II. Magister en Ciencias Internacionales y Diplomacia; Ingeniera en Marketing y Negociación Comercial; Universidad de Guayaquil, Guayaquil, Ecuador.

III. Magister en Administración de Empresas mención en Negocios Internacionales; Ingeniero Comercial; Universidad de Guayaquil, Guayaquil, Ecuador.
} 


\section{Resumen}

El propósito general que se dio a esta investigación, conllevó al análisis del proceso de control y seguimiento de contenedores liberado del almacén del puerto de Guayaquil. La metodología empleada fue la investigación descriptiva de los conglomerados empresariales en movilización de contenedores, donde se determinó como objetivo general: Desarrollar un software en seguimiento y control de mercancías dentro del contenedor. La muestra se derivó de los empresarios que generan el servicio de movilización del contendor al ser liberado de las almaceneras y de los contratiempos que el dueño de la mercancía mantuvo por mucho tiempo, siendo el precinto electrónico no tan seguro como se lo planteo hace décadas atrás, para la recolección de datos, se utilizó un cuestionario conformado por 5 preguntas. Se concluyó que el tipo de cinto electrónico, sería la solución del sistema de seguimiento y control de contenedores, pero ante el fracaso que este genera y no complementa el $100 \%$ de éxito, acorde a estos pormenores se genera un software de control y seguimiento en beneficio del empresario y dispositivo de ayuda de protección de mercancías que provee el precinto electrónico.

Palabras claves: Contenedores; almacenes de puerto; logística; sistema tecnológico; transporte de carga pesada; rutas. 
Procesos logísticos en el seguimiento de contenedores por vía terrestre desde la liberación de las almaceneras

\begin{abstract}
The general purpose of this investigation led to the analysis of the process of control and monitoring of containers released from the warehouse of the port of Guayaquil. The methodology used was the descriptive research of the business conglomerates in container mobilization, where it was determined as general objective: To develop software in monitoring and control of merchandise inside the container. The sample was derived from the entrepreneurs who generate the service of mobilization of the container to be freed of the warehouses and the setbacks that the owner of the merchandise maintained for a long time, being the electronic seal not as safe as it was raised decades ago, for data collection, a questionnaire composed of 5 questions was used. It was concluded that the electronic belt type would be the solution of the system of monitoring and control of containers, but before the failure that generates and does not complement the $100 \%$ of success, according to these details a control and monitoring software is generated in Benefit of the employer and device of aid of protection of merchandise that provides the electronic seal.
\end{abstract}

Key words: Containers; port warehouses; logistics; technology system; transporting heavy loads; routes. 


\section{Resumo}

O objetivo geral desta investigação levou à análise do processo de controle e monitoramento de contêineres lançados no armazém do porto de Guayaquil. A metodologia utilizada foi a pesquisa descritiva dos conglomerados comerciais na mobilização de contêineres, onde foi determinado como objetivo geral: desenvolver software em monitoramento e controle de mercadorias dentro do contêiner. A amostra foi derivada dos empreendedores que geram o serviço de mobilização do contêiner para serem libertados dos armazéns e os contratempos que o proprietário da mercadoria manteve por um longo tempo, sendo o selo eletrônico não tão seguro como foi levantado há décadas , Para a coleta de dados, foi utilizado um questionário composto por 5 questões. Concluiu-se que o tipo de correia eletrônica seria a solução do sistema de monitoramento e controle de recipientes, mas antes da falha que gera e não complementa o $100 \%$ de sucesso, de acordo com esses detalhes, um software de controle e monitoramento é gerado em Benefício do empregador e dispositivo de ajuda à proteção de mercadorias que forneçam o selo eletrônico.

Palavras-chave: Containers; armazéns portuários; logística; sistema de tecnologia; transportando cargas pesadas; rotas. 
Procesos logísticos en el seguimiento de contenedores por vía terrestre desde la liberación de las almaceneras

\section{Introducción.}

El Puerto Marítimo de Guayaquil, se ha convertido en uno de los centros portuarios más importante de Ecuador, posición que ha ganado gracias a las condiciones geográficas y características de las costas, los cuales en los últimos tiempos han desarrollado cambios significativos en lo que respecta a la infraestructura, con el objetivo de mejorar los procesos y tener mayor fortaleza interna, el estudio conllevó a generar verificación que el precinto electrónico no ha generado la seguridad esperada de la mercancía que se transporta una vez liberado el contenedor de la almacenera, por este efecto, los autores identificaron que las tecnologías en la actualidad aportan a múltiples empresas soluciones específicas que han generado resultados favorables, en base a estos parámetros la idea de un software de seguimiento de contenedores y de seguridad de la mercancía.

Lo antes expresado, orientó a los autores a verificar los pormenores que se deban plantear y lograr verificar la mejor estrategia tecnología a aplicarse y que genere además del servicio de seguimiento y vigía del contenedor con mercancías, que reduzca las pérdidas de producto y aumente la rentabilidad del empresario, la rentabilidad de un negocio en la actualidad que sea de provecho para muchos empresarios y a su vez genere el aporte sustancial a la población de educación superior, que se encuentra en busca de nuevas ideas de aprovechamiento de la tecnología en los tiempos actuales.

La metodología: es descriptiva, analítica y estructural. Los resultados de este análisis, se establecieron en base a poder generar un sistema tecnológico en aprovechamiento de las tecnologías de la investigación (TIC’s) que favorezcan a las empresas que necesitan además del cinto de seguridad en cada contenedor un sistema que genere una segunda alternativa de confianza que 
permita extender el sistema actual para poder verificar en tiempo real donde se encuentra el contenedor.

La Discusión conlleva al análisis de la participación de opciones estratégicas en base a los resultados de campo, para definir de forma concreta el planteamiento que establezca el soporte necesario para que la propuesta genere su validez. Se concluye en que un tipo de cinto electrónico, sería la solución del sistema de seguimiento y control de contenedores, pero ante el fracaso que este genera y no complementa el 100\% de éxito, acorde a estos pormenores se necesita de un software de control y seguimiento que ayude al cinto electrónico en el seguimiento y control de la mercancía a través de esta herramienta como estructura de carga de gran volumen pesada.

Como Objetivo General se originó el Desarrollar un software en seguimiento y control de mercancías dentro del contenedor

Entre los Objetivos específicos, se generaron los siguientes: a) Examinar los gastos por procesos logísticos aplicados a las empresas aduaneras en el seguimiento de contendores de los puertos secos del territorio ecuatoriano. b) Determinar qué tipo de herramienta tecnológica conllevaría a la innovación de los sistemas de control de contenedores de traslado desde el puerto marítimo al puerto seco. c) Identificar las falencias que mantiene el precinto electrónico ante el seguimiento y control de mercancías trasladadas en contendores.

\section{Métodos y materiales:}

Entre los métodos se generan los siguientes:

La determinación de Operacionalización de variables, conlleva a los investigadores a generar los métodos de estudio tales como: 
Procesos logísticos en el seguimiento de contenedores por vía terrestre desde la liberación de las almaceneras

- Método Exploratorio. - La intervención de la investigadora en la indagación previa en el área de estudio, conlleva a verificar que una vez se libera el contenedor como se está logrando generar un sistema de control en base al seguimiento del contenedor desde el puerto marítimo internacional y una vez haya llegado como este procede al seguimiento y control a nivel nacional (1)

- Método inductivo. - para lograr conseguir información en tiempo real, para de esta forma lograr inducirlos a participar dentro del estudio y así poder lograr conseguir la información en base al problema que les está afectando ${ }^{(1)}$

Acorde al tipo de materiales necesarios en este estudio, se establecen las herramientas necesarias para poder generar el mismo estudio.

- Entrevista: Una vez inducido a los posibles participantes del estudio, se procede al tipo de selección del mismo, donde se han intervenido a empresarios de compañía de transporte de contenedores de la ciudad de Guayaquil.

- Encuesta. - El resultado de las encuestas, se genera por un cuestionario a través de cinco preguntas estratégicas, las mismas que bajo la escala de LinkedIn se establece en cinco ítems, del cual bajo la tabulación se generan los valores por porcentajes y el respectivo gráfico donde se presentan los respectivos análisis del desarrollo en su fase pre-final ${ }^{(1)}$

\section{Logística de carga de contenedores tras la liberación a cargo del empresario en la almacenera}

\section{en el área de aduanas}

El tema aduanero, en base al crecimiento acelerado del comercio internacional, la integración de bloques comerciales, la transnacionalización de la producción, el desarrollo acelerado de la tecnología de la información y la revisión del papel del Estado y del sector privado ante las nuevas 
realidades sociales y económicas, son tendencias mundiales que influye en la necesidad del cambio en la actuación de los Sistemas Aduaneros y en la dirección de dicha innovación ${ }^{(2)}$.

El flujo de las mercancías, a través de vehículos pesados, genera muchas preocupaciones a cargo del gerente de la empresa o empresario de forma directa, siendo el factor primordial sobre el control y seguimiento del contenedor desde el puerto internacional y una vez llegue al puerto nacional, y realizar todo el proceso legal de declaración y liberación del contenedor con dicha mercadería a los diferentes puertos secos de destino final ${ }^{(3)}$.

En este punto la mayor parte del desarrollo de las regiones denominadas como puertos secos, se encuentran intercomunicadas gracias a las tecnologías de la comunicación por las redes de carreteras. Sin embargo, debido a la falta de control y desarrollo tecnológico en los puntos sin acceso a conectividad de control, conlleva a verificar si el proceso de control y desarrollo vial se está generando de forma correcta y si dentro de estos puntos de peligro no pase nada con el contenedor y la mercancía pueda desaparecer ${ }^{(4)}$.

Ante los parámetros antes establecidos, la logística estructural, administrativa y de suministro, conlleva a favorecer a los aspectos educacionales en los tiempos modernos, estos sumados a lo que la ética profesional establecida en la biología del ser vivo, se relacionan y establecen estrategias únicas en el campo educacional bajo el aprovechamiento del avance tecnológico en la actualidad ${ }^{(5)}$.

\section{Logística de transporte de carga pesada.}

En el campo económico, la aplicación de los cuatro sectores de importancia: logística, medio ambiente, empresa y transporte, establecen los factores necesarios para poder determinar si la 
Procesos logísticos en el seguimiento de contenedores por vía terrestre desde la liberación de las almaceneras

factibilidad del proceso de aplicación del sistema tecnológico de origen simplificado pueda o no generar los resultados esperados ${ }^{(6)}$.

El transporte proporciona dos servicios: movimiento físico y almacenamiento. El aspecto del movimiento físico es obvio. Se proporciona almacenamiento porque la carga se guarda durante el transporte del viaje, ya sea días, semanas o a veces hasta meses. Es práctica común hacer el transporte más lento y así tener la carga en almacenamiento por un poco de más de tiempo. Esto se hace comúnmente con el transporte marítimo de carga voluminosa, en el cual una entrega adelantada simplemente significa que a la carga necesita ser almacenada en tierra firme ${ }^{(7)}$.

El transporte puede ser visto como un medio para un fin, es decir, la forma en que se hace la logística, pero es también por sí mismo una industria importante. El transporte, incluyendo el movimiento de pasajeros, tradicionalmente ha representado un porcentaje muy interesante dentro de la conformación del Producto interno Bruto (PIB), pero esta participación se ha ido reduciendo debido al uso más eficiente de los diferentes medios ${ }^{(3)}$.

En muchas compañías hay puesto para quien se encarga del transporte, que se denomina administrador de transporte. Una descripción de esta posición nos da una idea de lo que es el planteamiento del transporte. El planteamiento del transporte, significa la selección del modo que, se va a utilizar, bajo la comprensión de tres principales claves:

- Velocidad: Es la capacidad de ir desde el origen hasta el destino tan rápido como se pueda.

- Consistencia: Es la capacidad de hacer que los envíos lleguen siempre al mismo tiempo. Una consecuencia importante de la consistencia son los requerimientos de inventario: entre más consistente es el transporte, menos existencias se necesitan. 
- Control: Es la capacidad de hacer cambios antes y durante el transporte. Las telecomunicaciones han revolucionado el transporte; ahora uno puede comunicarse con el conductor y cambiar la ruta si es necesario.

Los modos son combinaciones de redes, vehículos u operaciones. Incluyen, además de caminar, el coche, el sistema de carretera, los ferrocarriles, el transporte marítimo (barcos, canales y puertos) y el transporte aéreo (aeroplanos, aeropuertos y control de tráfico aéreo) ${ }^{(7)}$.

Motivaciones históricas, antropológicas y biológicas, conllevaron al hombre a desarrollar los diversos medios de transporte que existen en la actualidad. Pero también la curiosidad, característica del ser humano, lo ha incentivado a querer explotar su morada. (CASTELLANOS, 2015).

El transporte y la distribución son actividades logísticas de especial consideración para las empresas, ya que representan las dos terceras partes del conjunto de los costos logísticos. Además, estas actividades son clave dentro del concepto de servicio al cliente, ya que producen las entregas en el tiempo y la forma acordada, teniendo en cuenta las expectativas y requerimientos de este ${ }^{(7)}$.

La correcta gestión del transporte por liberación del contenedor en las almaceneras y la distribución de las mercancías por vía terrestre, lleva consigo una serie de decisiones que repercuten sobre el servicio y los costos, tales como el tipo de transporte utilizado en las importaciones y exportaciones (avión, carretera, barco, el servicio intermodal, etc.) ${ }^{(9)}$, el trazado de rutas, uno de los aspectos en los que más se ha trabajado en investigación operativa con el soporte de medios informáticos, los métodos de reparto, los medios de pago, las modalidades de descarga, etc. ${ }^{(11)}$

La distribución física Internacional (DFI), es un componente vital de toda operación internacional, que repercute en el campo de comercio de mercancías a nivel nacional, este puede 
Procesos logísticos en el seguimiento de contenedores por vía terrestre desde la liberación de las almaceneras

llegar a determinar el éxito o fracaso de una transacción debido a los costos y tiempos asociados con dicho proceso ${ }^{(8)}$. El porcentaje de costo de la DFI en el precio es variable, de acuerdo con el producto, destino de la exportación, peso, empaque, embalaje, etc., y puede variar entre un $10 \%$ hasta un $80 \%$ del valor del producto. Los modelos que se suelen usar para determinar los costos que se derivan de la DFI no consideran factores de gran relevancia como la inestabilidad continua de algunas variables, tal es el caso de la tasa de cambio, con lo cual se dificulta la realización de pronósticos de costos asociados a la DFI, y a su vez aumenta la incertidumbre en la determinación de costos de exportación de bienes en las organizaciones ${ }^{(7)}$.

\section{Logística de carga de contenedores}

El hablar de logística conlleva a verificar el tipo de procesos que este conlleva dentro de los aspectos de carga nacional. Esto lleva a establecer que el objetivo de la logística de carga nacional es parte de la satisfacción de la demanda y esto se debe ver reflejado en las mejores condiciones del servicio, costo y calidad ${ }^{(10)}$. La logística se encarga de la gestión de los medios necesarios para alcanzar este objetivo, movilizando el recurso humano necesario y el recurso financiero adecuado.

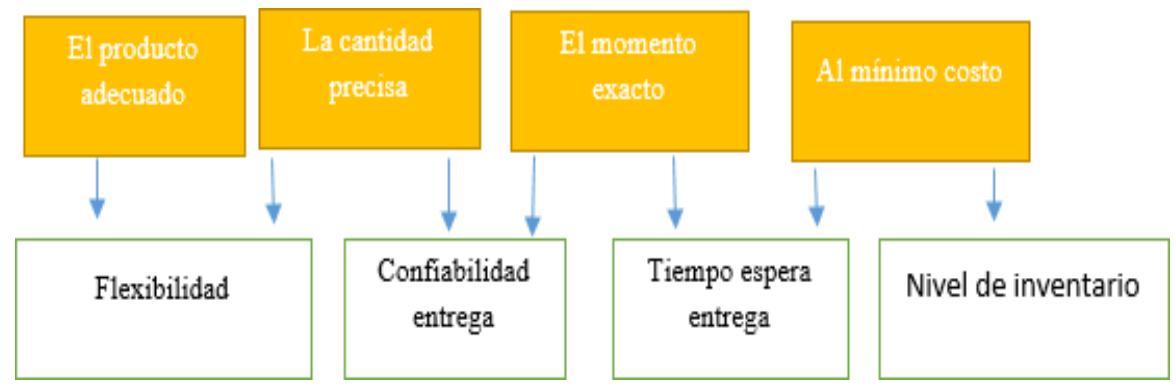

Nota: Figura 1. Objetivos y metas de la logística de carga nacional. ${ }^{(16)}$ 
Al implementar estrategias en especial en el transporte terrestre, este debe pasar por una serie de controles, desde la subida de carga, la declaración ante el UPC o tenencia política de la ciudad de destino de o embarque, registro de la mercancía (aspectos legales, como factura, declaración de impuestos, etc.), acorde al gráfico anterior si se cumplen los reglamentos logísticos como los antes mencionados y los cuatro ejes expuestos, se logrará la satisfacción del cliente y la empresa obtendrá reducción de costos de la cadena, que es uno de los factores por los cuales las organizaciones se enfocan en la logística ${ }^{(12)}$.

\section{Importancia de la logística estructural en el coste de movilización física de mercancías ${ }^{(3)}$}

El transporte de mercancías es una función de extrema importancia dentro del mundo de la distribución, ya que en él están involucrados fundamentalmente tres aspectos básicos: la calidad de servicio que se brinda a los clientes, los costes añadidos al producto de difícil recuperación y las inversiones de capital requeridas.

La función de trasporte está relacionada con diferentes aspectos, tato desde el punto de vista jurídico (legislación aplicable), como técnico-económico (tecnologías disponibles) o logístico. En este punto se debe centrar la atención fundamentalmente en los aspectos, técnicos, económicos y logísticos, dando solo una pequeña pinceladas delos aspectos jurídicos y reglamentarios para poder centrar el problema. ${ }^{(13)}$.

Aunque la palabra trasporte, se relaciona inevitablemente con el concepto de movimiento físico del producto, sin embargo, conviene desde este momento hacer algunas puntuaciones. El denominado transporte o tiempo de transporte, no se refiere solo al transporte físico del producto (mercancías de transito), sino al periodo comprendido desde que la mercancía está dispuesta en los muelles para su carga hasta el producto físicamente es descargado en el lugar de destino, lo cual 
Procesos logísticos en el seguimiento de contenedores por vía terrestre desde la liberación de las almaceneras

incluye necesariamente conceptos tales como; tiempos de espera Carga/Descarga de vehículos, paros en ruta, trasbordos, etc.

A complejidad en el mundo de distribución física, las diferentes tecnologías aplicables, las exigencias del servicio y una legislación en constante evolución homologable a nivel internacional, hacen que este servicio consuma en tono a un $40 \%$ de los gastos de distribución por logística estructural.

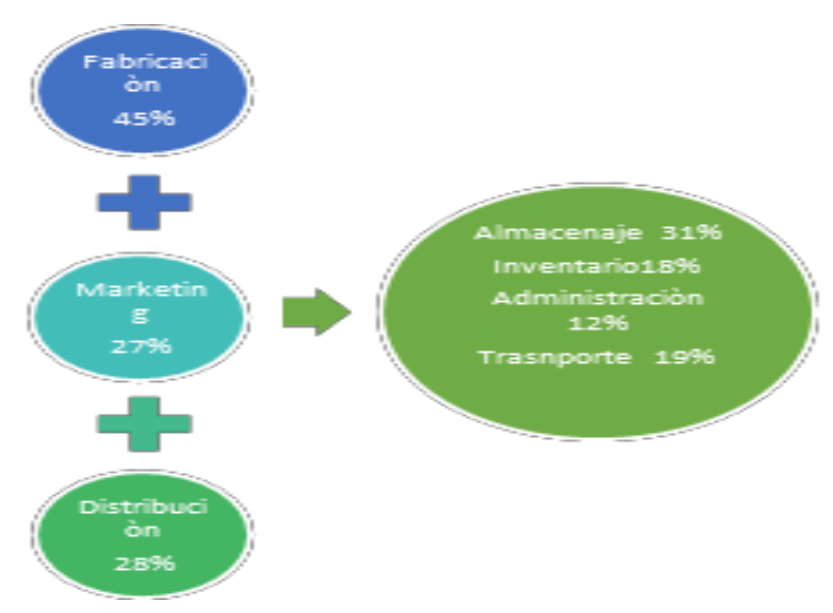

\section{Nota: Figura 2. Importancia de la logística estructural en el coste de movilización física de mercancías $^{(3)}$}

Como orientación se podría decir, que los referidos coste globales de distribución, se sitúan en torno a un $9 \%$ de las cifras de ventas, con una tendencia a la baja desde los últimos años, precisamente como consecuencias de las acciones logísticas emprendidas por las empresas más punteras en los sectores analizados, por lo que es fácil deducir que en muchos sectores empresariales estos costes se situarán en torno al $15 \%^{(13-14)}$. Estas cifras constituyen, evidentemente, un reto para las empresas, que deberían centrar su responsabilidad como gestores de este servicio en los siguientes aspectos fundamentales: 
- Utilización eficiente de los vehículos, así como del personal ligado a los mismos.

- Conseguir la máxima rapidez y fiabilidad en las entregas con un funcionamiento eficiente de la flota de transporte.

- Mantener la máxima seguridad tanto en el tráfico como en los productos que se transportan.

- Trabajar con la máxima flexibilidad, siempre de acuerdo con la legislación vigente.

En definitiva, se puede indicar que, los objetivos básicos, que la función de transporte desde un punto de vista logístico, se centran fundamentalmente en dos aspectos: dar plena satisfacción a los clientes en los términos de rapidez de entrega y minimizar los costes de la gestión a un nivel aceptable. Los nodos logísticos con puntos de intercambio de mercancías donde se realizan actividades que aportan valor añadido a la cadena logística de transporte, tales como la manipulación, el procesamiento y en su caso, el almacenamiento de la mercancía.

\section{Equivalencias por modo de transporte y adecuación de la carga}

Los diferentes modos de transporte han sido diseñados $\mathrm{y}$ acondicionados para la movilización de los distintos tipos de carga. Por ello, los operadores de los modos de trasporte han desarrollado varias fórmulas relativas a la relación volumen-peso, con base en la forma de los vehículos, los estudios económicos sobre la capacidad de carga de las bodegas o plataformas y los costos de operación por servicio de transporte de contenedores con mercancías ${ }^{(15)}$.

En la adecuación de carga que se va a transportar se deben tener en cuenta ciertos aspectos a fin de maximizar el espacio disponible en los sistemas de transporte. En el apilamiento, las cajas deben organizarse de forma tal que la estabilidad del arrume sea consistente. La firmeza se puede obtener mediante el uso de Zunchos, esquineros u otros mecanismos de inmovilización, e 
Procesos logísticos en el seguimiento de contenedores por vía terrestre desde la liberación de las almaceneras

igualmente se recomienda trabar solo el último o los dos últimos tendidos del arreglo, teniendo en cuenta no exceder a altura máxima, la cual determina su diseño estructural.

$\mathrm{Al}$ hacer uso de un contenedor, mediante un contrato, se adquiere el compromiso de verificar y cuidar el equipo que se entrega en comodato y que queda bajo custodia durante el periodo que estipule e acuerdo por consiguiente, se asumen las responsabilidades y la administración de los riesgos a fin de evitar que sea utilizado por organizaciones y la administración de los riesgos a fin de evitar que sea utilizado por organizaciones delincuenciales para el transporte y el tráfico ilegal ${ }^{(4)}$.

De esta forma, el comodante debe recibir el contenedor en condiciones aptas para que sea utilizado con el llenado de las mercancías y su posterior transporte. Por ello debe llevar a cabo una inspección y/o revisión, a fin de determinar las condiciones o daños que presente y que puedan generar un riesgo o reduzcan las condiciones de eficiencia del contenedor.

De aquí parte el interés de todos los involucrados en el proceso por asegurar que, en cada contenedor, se lleven a cabo las inspecciones apropiadas que permitan certificar que el equipo y las reparaciones que se le hayan realizado cumplan con las condiciones de calidad exigidas. Estas revisiones se deben efectuar tanto al momento de recibir el contenedor, como a la entrega de este, teniendo en cuenta verificar la parte exterior y la interior ${ }^{(17)}$.

Antes de comenzar con la estiba del contenedor es necesario conocer que los límites de peso de este se rigen bajo la norma estándar internacional ISO 668, la cual establece que el peso bruto permisible para la mayoría de los contenedores estándar de $20 `$ y 40`, es de $30.480 \mathrm{~kg}$. Aunque alguno contenedores más recientes tienen un peso bruto mayor ${ }^{(18)}$. 
Además, es necesario contar con información precisa del embalaje, peso y dimensiones de la carga, así como también de las dimensiones internas del contenedor y sus restricciones, con el fin de proceder a elaborar el plano de estiba que permita optimizar la utilización de la capacidad del contenedor, simplificar el material de amarre necesario. ${ }^{(7)}$.

Una vez estibado el contenedor, se debe tener en cuenta que la carga depositada, si viaja en camión o tren, estará sujeta a movimientos bruscos y a curvas que sacudirán la mercancía. Si se transporta por barco, las olas del mar causaran movimientos a la carga hacia los lados, hacia arriba y hacia abajo, por lo cual esta debe estar bien afianzada en el contenedor, esto es lo que se le llama tensión mecánica ${ }^{(22)}$.

Con el fin de amarrar la carga firmemente, los contenedores han sido diseñados especialmente para ello; en su estructura cuentan con facilidades que ayudan al manejo, manipulación y aseguramiento de las mercancías ${ }^{(18)}$.

\section{Infraestructuras e instalaciones de seguridad del transporte del contenedor}

Las infraestructuras e instalaciones para el transporte se constituyen en uno de los bastiones fundamentales e imprescindibles para el desarrollo y evolución de esta importante actividad económica y social entre empresarios de países. Su mejoramiento y perfeccionamiento tiene una influencia directa sobre las condiciones y la calidad de vida de sus ciudadanos, debido a que les proporciona un mejor acceso a numerosos servicios y favorecen una repartición más equitativa de la renta y la riqueza ${ }^{(23)}$.

Igualmente contribuyen con el mejoramiento de la competitividad, ya que permiten el incremento de os niveles de productividad del trabajo y del capital privado; al mismo tiempo, 
Procesos logísticos en el seguimiento de contenedores por vía terrestre desde la liberación de las almaceneras

propician un crecimiento económico estable y sostenido y sientan las bases para una mayor equidad regional. Por ello, las infraestructuras e instalaciones se transporte se constituyen en elementos esenciales para la articulación y cohesión de los territorios y hacen que el desarrollo de ellas es objeto de atención creciente por parte de los gobiernos ${ }^{(20)}$.

Con el trascurrir del tiempo, los puertos, aeropuertos, terminales ferres y de camiones han ido superando su misión inicial de satisfacer las necesidades de los pasajeros y las mercancías en el tránsito entre los modos de transporte marítimo, aéreo y terrestres para convertirse en verdaderos centros empresariales, logísticos, de negocios; en polos de desarrollo, cuya influencia trasciende los límites estrictos de sus recintos ${ }^{(16)}$.

En otras palabras, su importancia es tal que estas infraestructuras no solo representan la movilización de tráfico internacional de carga de pasajeros, sino que adquieren un carácter estratégico para diferentes sectores económicos en el ámbito nacional, y resultan, además, esenciales para las comunicaciones entre los países y sus diferentes regiones ${ }^{(5)}$.

En este orden de ideas, por ser el marítimo el principal sistema de transporte, pues moviliza la mayor porción de cargas del comercio internacional, los puertos se constituyen en nodos importantes de las cadenas de transporte internacional; además, son puntos de paso obligado y, en muchos caos, fronteras en las que se produce una detención de las mercancías por razones aduaneras, o bien por causas comerciales o fiscales ${ }^{(24)}$.

Estas circunstancias hacen de los puertos puntos relevantes de las cadenas logísticas, cuyo objetivo es mejorar la circulación de las mercancías, tanto en la fase de aprovisionamiento (materias primas y equipos) como es las de producción (productos semielaborados) y distribución (productos acabados) ${ }^{(25)}$. 


\section{Sistema de transporte terrestre de contenedores}

El transporte en camiones es una parte indispensable de cada envió nacional o internacional de mercancías por contenedores.es un importante eslabón en la cadena intermodal, pero también en algunas regiones se usa para el intercambio comercial internacional ${ }^{(34)}$. Su papel en el intermodalismo es unir a un puerto marítimo o la estación vial de carreteras ya sea por ferrocarril o camiones, tráiler con el punto local o destino final. En otras palabras, la mercancía es cargada y descargada en las instalaciones del consignatario o del destinatario, no en el puerto (26). Los camiones llevan a algunos embarques internacionales $y$, por supuesto, embarques que no son intermodales.

El papel de transporte de contenedores en camiones varía dependiendo de la región; áreas escasamente habitadas, donde el ferrocarril u otro medio terrestre de transporte deben estar muy bien establecidos los camiones se usan más para entregas locales, pero los tráileres, son de transporte a larga distancia sirven en vías no muy bien posicionadas, en el área de paso lateral donde no se perjudique al mercado o área densamente poblada, estos pasos son realmente útiles ${ }^{(33)}$.

Todos los medios de transporte están influenciados por las regulaciones. Para el caso del transporte terrestre, los reglamentos locales marcan sus directrices y establecen límites de velocidad, registro, tonelaje máximo que se puede transportar y otros más. Lo anterior significa que entre más largo sea el viaje, mayor cantidad de trámites hay que realizar, lo cual crea una fuerte desmotivación por este tipo de transporte. Mientras que un medio como el marítimo no requiere de tantas formalidades ${ }^{(29)}$.

En cuanto a los contenedores, esto gracias al precinto de seguridad, además de las regulaciones y cumplimientos legislativos que el camión de carga de gran volumen pesada, debe 
Procesos logísticos en el seguimiento de contenedores por vía terrestre desde la liberación de las almaceneras

considerar, también el contenedor debe estar resguardado además del precinto electrónico, con otra tecnología que conlleve a verificar la seguridad, seguimiento y control de tiempos en el traslado de mercancías desde el puerto marítimo al puerto seco o viceversa ${ }^{(27)}$.

La importancia del transporte multimodal en las cadenas de suministro se debe analizar detenidamente, por un lado, se beneficia de las virtudes que otorga la conexión directa: origendestino, y, por otro, se puede considerar tan fuerte como el más débil de los sistemas que lo conforman. De aquí el porqué de tener modos de transporte bien estructurados, unas buenas redes y sistemas de integración física entre los mismos, mediante infraestructuras como las terminales especializadas, además, contar con el apoyo de una buena base institucional ${ }^{(28)}$.

La implementación del transporte multimodal que trasladen contenedores de $20^{`}$ y $40^{`}$ empieza a impactar las cadenas logísticas por las economías de escala que conllevan a que los puertos especialicen y actualicen sus instalaciones, pues tendrán que manipular mucha más carga que un puerto de carga granel o en bultos ${ }^{(6)}$. Desde el punto de vista de la logística, la importancia radica en la competitividad y, viéndose así, los exportadores e importadores, principales usuarios de los puertos, deben ajustar sus cadenas de suministro a estas nuevas condiciones del mercado, ya que el éxito de una empresa lo determina no la calidad de sus productos, arcas o precios, sino el transporte de bienes, es decir, llevarlos desde su punto de origen hasta las estanterías del comprador. Esto va a ser, o es más importante que cualquier otra estrategia. Hacer alianzas con empresas, cuyas fortalezas de negocios sean la debilidades de otros es la mejor práctica ${ }^{(13)}$.

El transporte de mercancías es una actividad económica compleja que permite y facilita el transporte de desplazamiento de carga de un origen a un destino, para lo cual integra tres elementos que se interrelacionan entre sí: la infraestructura, los medios o vehículos y el servicio mismo ${ }^{(32)}$. 
Lo anterior explica en cierto modo, la razón del crecimiento de los terminales de contenedores a través del mundo; cada exportador o importador, buscando esta competitividad, decidió utilizar el manejo de contenedores para sus productos con el propósito de trasladar estos beneficios a los estados financieros de sus compañías, lo cual impulso a los puertos a rediseñar sus estructuras con el fin de atender estas grandes cantidades de mercancías en sus instalaciones ${ }^{(7)}$.

La cadena de suministro y su administración adquieren gran importancia dentro del transporte multimodal, ya que allí confluyen varios de los actores de esta cadena logística, en la cual existen disimiles ramificaciones. Por ello, se debe tener especial cuidado al momento de negociar.

El flujo de las mercancías, a través de vehículos pesados, genera muchas preocupaciones a cargo del gerente de la empresa o empresario de forma directa, siendo el factor primordial sobre el control y seguimiento del contenedor desde el puerto internacional y una vez llegue al puerto nacional, y realizar todo el proceso legal de declaración y liberación del contenedor con dicha mercadería a los diferentes puertos secos de destino final ${ }^{(30)}$.

En este puto la mayor parte del desarrollo de las regiones denominadas como puertos secos, se encuentran intercomunicadas gracias a las tecnologías de la comunicación por las redes de carreteras. Sin embargo, debido a la falta de control y desarrollo tecnológico en los puntos sin acceso a conectividad de control, conlleva a verificar si el proceso de control y desarrollo vial se está generando de forma correcta y si dentro de estos puntos de peligro no pase nada con el contenedor y la mercancía pueda desaparecer ${ }^{(31)}$. 
Procesos logísticos en el seguimiento de contenedores por vía terrestre desde la liberación de las almaceneras

\section{Resultados.}

Se generó encuestas a 48 participantes que trasladan contenedores con mercancía importada de forma legal dentro del territorio ecuatoriano.

$$
\begin{gathered}
N^{*} Z^{2} p^{*} q \\
=\frac{\square}{d^{2} *(N-1)}+Z_{\alpha}^{2} * p * q
\end{gathered}
$$

Se generó preguntas que identificaron la participación de la muestra en estudio y logró verificar los siguientes resultados.

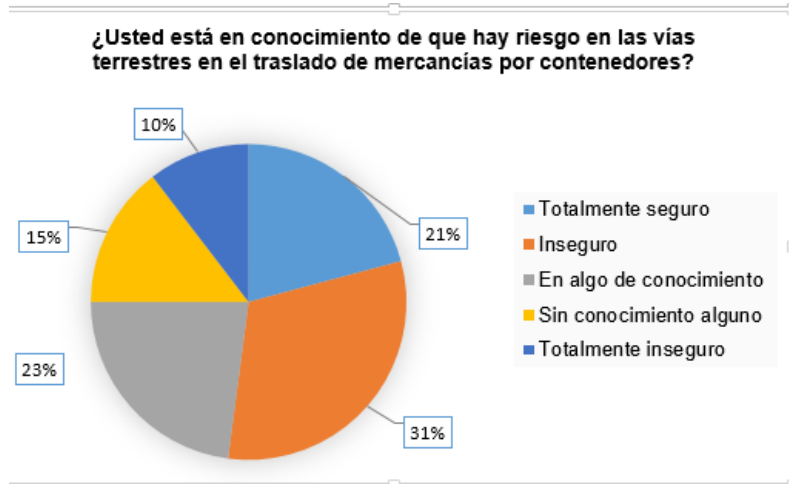

\section{Nota: Figura 3. Riesgo que se ubica en las vías por transportación bajo precinto electrónico}

del contenedor (Andrea Villacis, 2016)

Análisis: Los empresarios, de los cuales se involucran los trasportistas, como responsables de los contenedores, empresarios importadores y exportadores, y comerciantes que son consolidadores externos para poder exportar, ellos indican en un $21 \%$ que está en total seguridad que hay riesgos en la vía donde se transportan lo contenedores con mercancías, el $31 \%$ indican estar inseguros ya que a ellos no se les han presentado irregularidades en el transporte, el $23 \%$ indican que en este tipo de casos mantienen algo de conocimiento, el $15 \%$ indican que como no ha sucedido 
nada malo en las vías terrestres, ellos concebían al idea de seguridad absoluta de sus mercancías y el $10 \%$ indican estar totalmente inseguros

$¿$ Cree usted que el software de seguimiento utilizado en la actualidad por su empresa, es el que le brinda mayor seguridad en el seguimiento y control del contenedor con mercancía?

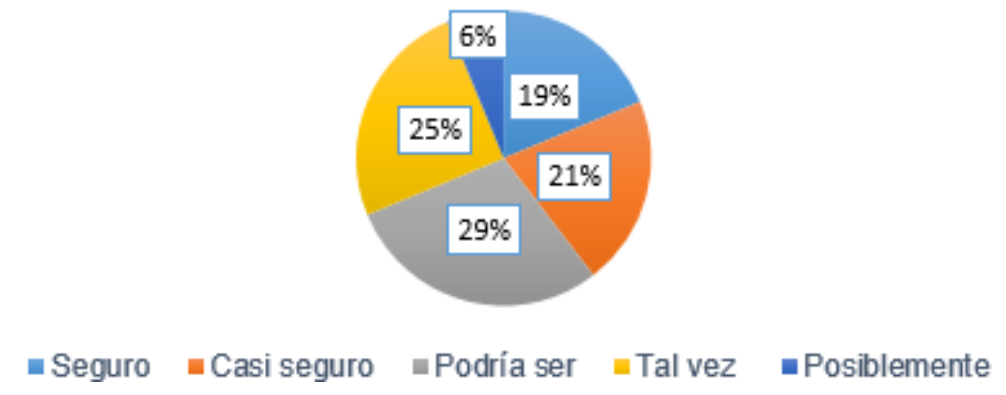

Nota: Figura 4. Tipo de software de seguimiento utilizado en la actualidad. (Andrea Villacis, 2016)

Análisis: Los empresarios intervenidos, en su aporte indican bajo la pregunta 2, que el software utilizado en un $19 \%$ genera total seguridad en base al sistema de seguridad de traslado de control y seguimiento del contenedor, el $21 \%$ indican estar casi seguros, el $29 \%$ indican que podrían ser y el 25\%indican que tal vez y el 6 indican posiblemente. 
Procesos logísticos en el seguimiento de contenedores por vía terrestre desde la liberación de las almaceneras

¿Ustedes han verificado problemas luego de liberar el contenedor con mercancias, en el traslado del mismo al punto final de entrega?

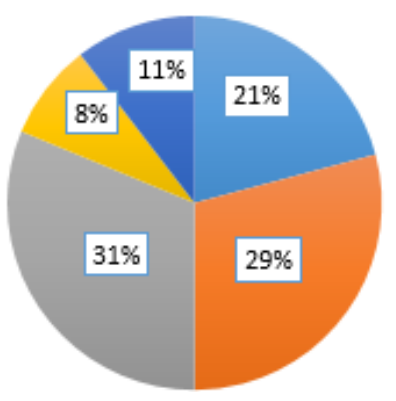

- Muchos problemas

- Pocos problemas

- Ningún problema

« Sin verificar

- No mantenemos problemas

\section{Nota: Figura 5. Problemas en la liberación de mercancías para efectuar el traslado del}

contenedor al centro del país. (Andrea Villacis, 2016)

Análisis: En base al seguimiento y control del contenedor, los entrevistados indicaron que presentan muchos problemas en un $21 \%$, el $29 \%$ indican que mantienen pocos problemas, el $31 \%$ indican que no mantienen ningún problema, el $8 \%$ indican que no han podido verificar dicha situación y el $11 \%$ indican no mantener problema alguno. 


\section{¿Mantienen algún tipo de estrategia en base al seguimiento y seguridad del contenedor con mercancías?}
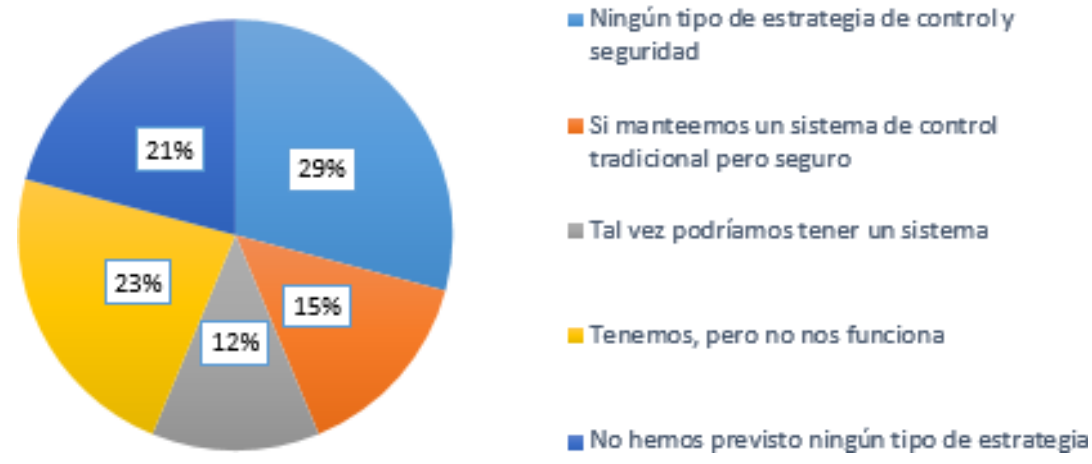

\section{Nota: Figura 6. Tipo de estrategia a plantearse en base al seguimiento y seguridad del} contenedor de mercancías en su traslado. (Andrea Villacis, 2016)

Análisis: En base si mantienen o no algún tipo de estrategia de seguimiento y seguridad, el $29 \%$ indico que no mantienen ningún tipo de estrategia, el 15\% indican que si mantienen un sistema tradicional que les brindad resultados y seguridad en su aplicación, el $12 \%$ indican que tal vez la tendrían pero no han verificado si funciona o no, el 23\% indican si mantienen una estrategia pero no les ha brindado resultados favorables y el $21 \%$ indican no haber previsto ningún tipo de estrategia para este fin. 
Procesos logísticos en el seguimiento de contenedores por vía terrestre desde la liberación de las almaceneras

$¿$ Cree usted que deben establecer los profesionales propuestas en

base a software de seguimiento y control que no afecte el sistema

por bloqueo en este caso montañas de la señal?

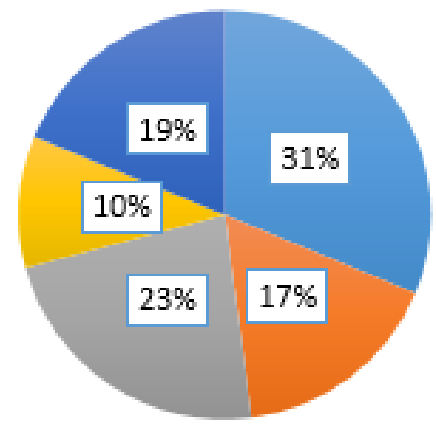

\author{
- Totalmente de acuerdo \\ - Ni de acuerdo ni en desacuerdo \\ Me interesa \\ - Tal vez \\ - No está dentro de mi planificación \\ empresarial
}

\begin{abstract}
Nota: Figura 7. Tipo de propuesta y su participación, concordante al tipo de profesionales que opten por establecer ideas en solución del problema. (Andrea Villacis, 2016)

Análisis: El grupo de profesionales especializados en esta área o competencia, el $31 \%$ indica estar totalmente de acuerdo, el $17 \%$ indica estar no de acuerdo i en desacuerdo, el $23 \%$ que le interesa, el 10\% indican que tal vez sería la solución, y el 19\% indican que no está dentro de la planificación de la empresa adquirir dicha herramienta tecnológica.
\end{abstract}

\title{
Discusión.
}

Acorde al estudio generado, se entiende que la logística como tal, es una herramienta que favorece cualquier actividad comercial, de esta forma los empresarios internos y externos indicaron en un $31 \%$ acorde al $100 \%$ de la encuesta estar inseguros cuando se libera el contenedor de puerto y se traslada la mercancía de un punto a otro, eso los llena de inquietudes, generando en muchos casos perdidas de mercancías a pesar de que el precinto electrónico este intacto. 
Según la opinión de estos empresarios, ellos indican ante la idea de generar un software que establezca dos parámetros se seguridad en el traslado de mercancía al punto de entrega final, ellos en un $29 \%$ tienen la esperanza de que esta sea la solución esperada.

A pesar del problema que se presenta por la desconfianza en el traslado de mercancías, los empresarios en un $31 \%$ indican no haber tenido problemas de perdida de mercancías y el restante lo indica como afirmativo, lo que implica que este es un problema que sobrepasa el entendimiento de cómo se puede violar el precinto electrónico y este al revisarlo llegue intacto al punto final de entrega de mercancía.

En cuanto a la seguridad y seguimiento del contenedor con la mercancía el $23 \%$ de los empresarios, indica haber mantenido estrategias aplicadas al sistema de seguridad, en muchos casos generó costos inesperados y en otros no dio resultado, del cual más del $65 \%$ no han generado ningún tipo de estrategias en base a este punto de entendimiento.

Los empresarios en la intervención de la autora de este estudio, generaron en su participación la afirmación de que el talento profesional de Guayaquil y de Ecuador, deberían plantear posibles soluciones a través de proyectos macro, donde este problema como un referente de traslado de mercancías se pueda establecer algún software que establezca la seguridad esperada y no incurran a perdidas eventuales de mercancías mermado la rentabilidad del negocio.

\section{Conclusiones.}

Los empresarios entrevistados, indican que la aplicación de un nuevo dispositivo electrónico interno en el contenedor, ha generado reducción de costos operativos e incrementado la rentabilidad del negocio, ante estos parámetros, los autores han podido socializar con el área de Aduana para 
Procesos logísticos en el seguimiento de contenedores por vía terrestre desde la liberación de las almaceneras

poder generar un tipo de promoción del software como ayuda para la vigía complementaria ante el cinto electrónico como único medio internacional/nacional de seguridad de contenedores con mercancías.

El precinto electrónico, fue un buen sistema desde sus inicios, pero ante la evolución y constante innovación tecnológica, se torna vetusto dicho sistema del cual se establece un software que complemente la seguridad de la mercancía transportada en el contenedor ya sean por importaciones o exportaciones en el Puerto Marítimo de Guayaquil

Indagar el beneficio y/o perjuicio que un sistema de control y seguimiento vetusto puede originar para las empresas aduaneras en la liberación y seguimiento de contenedores.

El tipo de cinto electrónico, sería la solución del sistema de seguimiento y control de contenedores, pero ante el fracaso que este genera y no complementa el $100 \%$ de éxito, acorde a estos pormenores se necesita de un software de control y seguimiento que ayude al cinto electrónico en el seguimiento y control de la mercancía a través de esta herramienta como estructura de carga de gran volumen pesada.

Se verificó el coste del proceso de control y seguimiento de contenedores, del cual existe constante incremento, que se aplican a los procesos logísticos aprovechados por las empresas importadoras y exportadoras, que trasladan sus mercancías por contenedor y a través de vehículos de transporte de carga pesada en diferentes sectores del territorio ecuatoriano.

\section{Bibliografía}

1. Alexa, A. (2012). Modalidades de Exportación en Colombia. Bogotá: Broker logistic Transpor.

2. Anaya, T. J. (2015). El Transporte de Mercancias. Madrid: Gráficas Dehon.

3. Arola; TARIC y AENOR. (2015). Guía para la PYME Exportadora: Aduanas, logística e inspección . España: AENOR. 
4. Ballesteros. (2011). Comercio Exterior, Teoría y Práctica. Barcelona: Trillas.

5. Castellanos, F.F. (2011). Direccionamiento estrategico de sectores industriales en Colombia a partir de sistemas de inteligencia tecnólogica. Bogotá: UNC.

6. Castellanos, R. Á. (2015). Logistica comercial internacional. Barranquilla: UNINORTE.

7. Cossio, JJ. (2013). Manual de Derecho Aduanero. Lima: Rodhas.

8. Costa, J. (2011). Trtatado de estiba. Bogotá: Estudios Nauticos Costa.

9. Cuartas, JJ. (2013). Emprendimiento de Base Tecnológica. Boston.

10. EMERALD GROUP. (2011). Quality deployment aids effective strategic desicion making, alining customer demands with future business plans. Boston: DOI.

11. Exterior, M. D. (2015). Plan Estrategico de Logistica Internacional. Guayaquil.

12. Farfaks. (2011). LIDERAZGO COMERCIAL. En Á. C. Ramirez, Logistica Comercial internacional (págs. 78-79). Barranquilla: UNINORTE.

13. García-lomas, O. L. (2015). Negociación Internacional. New York: Global Marketing Strategies.

14. Larrucea, Jaime y SAGARRA, Ricardi. (2013). Transporte en Contenedor. Madrid: Marge books.

15. Lassagne, T. (2015). Objetivos y metas de la logistica. En Á. C. Ramirez, Logistica Comercial Internacional (págs. 145-146). Barranquilla: UNINORTE.

16. Lavanda F. (2014). Negocios Internacionales. Arica, Chile: Brief.

17. Mullins, J. (2011). VOICES What to do before you write a business plan. New York: Strategy Review.

18. OMI. (2013). IMO Update Study for the GHG Emissions Estimate for International Shipping. Primer informe del Comite Directivo (pág. 66). Londres: MEPC.

19. ORGANIZACIÓN MUNDIAL DE ADUANAS, 2., \& ORGANIZACIÓN MUNDIAL DE ADUANAS. (2015). Administracion Aduanera. Londres.

20. Pradican. (2013). Manual sobre control de Contenedores. Perú: Secretaría de la CAN.

21. PROECUADOR. (2011). Guía Logística Internacional. Quito.

22. Rodriguez, J. M. (2014). Trasnporte de Mercancias por Carretera. Madrid: Marge Books.

23. Sampiere. (2014). Metodología de la investigación. México. Mc Graw Hill

24. Sánchez, S. M. (2014). Exportar es fácil. si sabes cómo. España: Ariel.

25. Sandoval, L. (2012). Logística Internacional de importaciones. Lima: ENA.

26. Sanzs, R. (2015). Actividad de Comercio, Inspección, Certificación de lecturas y casos. Valle de Coquimbo.

27. SENAE. (2012). Manual especifico para la Re-estiba de unidad de carga. Guayaquil. 
Procesos logísticos en el seguimiento de contenedores por vía terrestre desde la liberación de las almaceneras

28. SENAE. (2013). Guia de operadores de Comercio Exterior para el ingreso y salida de unidades de carga vacia desde y hacia zonas aduaneras. Guayaquil: SENAE-GOE.

29. Serrano, J. (2013). Trasnporte de Carga, una cuantia aún a medio pagar. Revista Logistica, 73-74.

30. SUNAT. (2014). Procedimientos de Carga, una cuantia aún a medio pagar. Revista Logistica, 73-74.

31. THE INTERNATIONAL CHAMBER OF COMMERCE. (2012). Guia básica internacional, Conocimientos para exportar e importar. Madrid: Camara de Comercio Internacional.

32. Ubal, P. (2014). Costos de Capacidad ociosa en transporte de contenedores. Londres: EAE.

33. UNCTAD. (2015). Conferencia de las Naciones Unidas sobre comercio y Desarrollo. El Transporte Marítimo (pág. 149). New York: NU.

34. UNTAC. (2011). EL Transporte Marítimo. Publicacion de las Naciones Unidas de Venta (págs. 125128). Nueva York y Ginebra: Library publication . 